\title{
Structural Model of a Training Computer Program for Improving Professional Skills of a Student in a Role of a District Polyclinic Physician
}

\author{
Sergey Bulatov ${ }^{1}$, Enzhe Kharisova ${ }^{1}$, Roman Lavrenov ${ }^{2}$, Bulat Abbyasov ${ }^{2}$, Evgeni Magid ${ }^{2, *}$ \\ ${ }^{1}$ Center for Practical Skills, Kazan State Medical University, 49 Butlerov Street, Kazan, Russian Federation \\ ${ }^{2}$ Laboratory of Intelligent Robotic Systems (LIRS), Intelligent Robotics Department, Institute for Information Technology and Intelligent Systems, \\ Kazan Federal University, 35 Kremlyovskaya Street, Kazan, Russian Federation
}

\section{ARTICLE INFO}

Article History

Received 04 November 2020

Accepted 17 May 2021

Keywords

Medical education

training computer program

physician skills

\begin{abstract}
An important feature of a medical education in the context of the COVID-19 pandemic is a migration of classes into an online or mixed format, which requires simulated-based teaching methods with elements of robotics and artificial intelligence. We analyzed computer programs for maintaining medical records of patients that are employed by various polyclinics of Kazan city. Based on the analysis, we summarized requirements for a training computer program that could develop medical records maintaining skills of university students. The proposed program focuses on modeling situations associated with pre-hospital stage processing of medical records for patients. The paper presents an improved architecture of the program and an example of its use.
\end{abstract}

(C) 2021 The Authors. Published by Atlantis Press International B.V. This is an open access article distributed under the CC BY-NC 4.0 license (http://creativecommons.org/licenses/by-nc/4.0/).

\section{INTRODUCTION}

The current epidemiological situation forced a transition of a higher education to distance learning in an online mode [1]. While a main pedagogical emphasis moved to information technologies applications for educational process, including computer programs and artificial intelligence [2,3], specifics of Medical Information Systems (MIS) do not allow all students to master them independently. A preliminary training of medical students, whose major is far away from IT and computer science, that could allow a smooth work with MIS used in practical health care has become acute.

The COVID-19 pandemic, which by the time of writing this paper has infected over 135 million people around the world and took over 3 million lives, has been a contributing factor in this process as it changed a traditional doctor-patient relationship and replaced face-to-face communication with a remote monitoring of patients $[4,5]$. In local polyclinics, computer programs create Electronic Medical Records (EMR) [6] of patients, draw up sick lists, monitor laboratory tests, and ensure preservation of information about patients' treatment. Moreover, while in the previous decades almost every local polyclinic had its own MIS structure, recently there is a clear trend toward unification of various MISs under a state umbrella, in a top-down fashion. In turn, medical universities are interested in preparing students for the future active work with

*Corresponding author. Email: magid@it.kfu.ru
MIS in the outpatient. In literature sources, the authors had found only isolated reports about special training of students to work with EMRs and MIS [7]. In this paper, we present an architecture and an interface of a training program, which repeats the main elements of an EMR.

\section{RELATED WORK}

In medical universities of Russia, a special training of graduate students to work with EMRs of a regional polyclinic is still experimental. Programs that are used in multi-specialty hospitals for inpatient patients might be used as educational materials in universities, e.g., EMR created in MIS "Aurora" for multi-specialty hospitals [8] provides a training interface "UMS University" for individual tasks, with a separate entrance for students and teachers, and an access to a server via the Internet.

In regional polyclinics of Kazan city EMRs vary within commercial MISs -BARS.MED, ERP-system, 1C: Medicine (Registry), and VITACORE. These systems have centralized databases with a secure access for each user through standard operating environments (Microsoft, Linux) and a Web browser. A number of short pilot projects of using a demo version of EMRs by BARS.MIS in the educational process in numerous cities of Russia was reported [9].

An example for using an educational EMR in a sub-internship was reported by Indiana University School of Medicine, USA [7]. It involved 16,602 graduated medical students in the period from 
2012 to 2016. The students reported a positive influence of this training on their knowledge expansion and future work. Some participants pointed out on having difficulties in a communication part with a patient. In turn, the authors of the program considered as one of the main difficulties a necessity of database updating with regard to decease treatment recommendations and guidelines. A positive effect of using EMR in training of higher medical schools' students was demonstrated in [10-12] as an important part of an education, while students, in turn, reported that mastering EMR had a positive role in preparing them for a future profession $[13,14]$.

Today, there are no explicit references in literature about any unified pedagogical approach of teaching medical students to work with EMR in Russia. We believe, the reasons are related to a commercial nature of such products, which prevents their free distribution, as well as an absence of a single EMR/MIS that is required for an obligatory use by a local government. Therefore, to facilitate medical education, local teams of educators create unique training computer programs that simulate real MIS behavior to support the necessary training. Main characteristics of such training programs (to provide an acceptable level of simulation) are their similarity to existing commercial products, a capability to evaluate student's actions and to help him/her during a task execution. This work, being an extension of our previous conference paper [15], presents an architecture and interfaces for the MIS training program for students of higher medical schools.

\section{PROGRAM ARCHITECTURE AND INTERFACES}

\subsection{Situation Modeling}

One of the goals of the program is to combine disparate information (patient data, proposed treatment and additional literature) within a single block to allow transforming theoretical knowledge into practical skills [15]. Collecting information from a patient and conducting an objective examination of the patient comprise a preliminary stage of work.

We model a typical real world situation as follows. Patient Ivanov Kirill, 51 years old, came with complaints of a fever, a cough, and a general weakness. The student's task is to create an EMR for the patient and determine a medical treatment plan. A student manually enters his name, date and time of a consultation, and a patient's name to open active fields, which consist of complaints at the time of examination, medical history, patient's life history, and examination data (Figure 1).

The stage ends with establishing a preliminary diagnosis and entering it in the corresponding field. The patient information is stored in the database and can be reused later by the student at the next stages.

\subsection{Analysis}

After making a preliminary diagnosis, in accordance with a treatment process algorithm, the patient should be assigned the necessary laboratory and instrumental assessment methods (Figure 2).

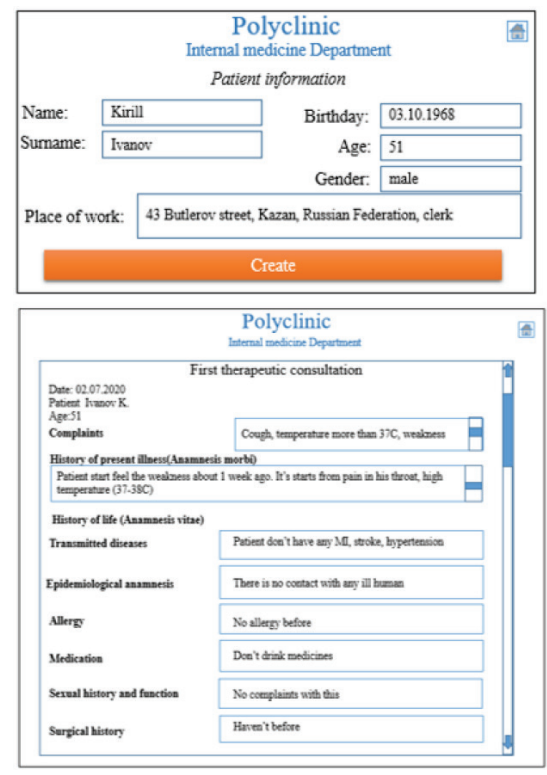

Figure 1 Creating of personal electronic medical card.

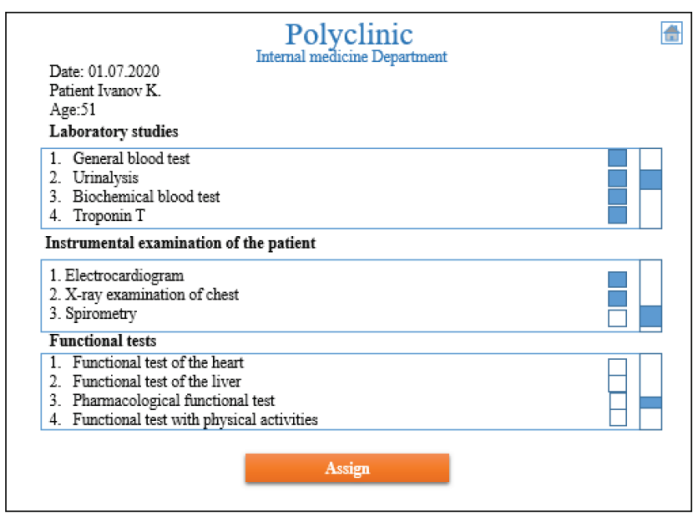

Figure 2 The lists of available laboratory and instrumental tests.

It is required to select an optimal (strictly regulated by current standards of the state) set of methods among proposed ones. The student selects tests using interactive buttons on the right side of the screen (in Figure 2 buttons that switched their color to blue are the selected ones, white color are the ignored options) and completes the process by clicking the assign button.

The program automatically evaluates the correctness of the tests' selection and informs the student with colored indicators on the screen: green color means a right decision, red color is for a wrong choice, yellow color marks a missed option. Figure 3 demonstrates the evaluation of the student's selection of the tests. The selection of the blood test to determine a level of the troponin $\mathrm{T}$ was a wrong choice, and the program highlighted it in red. The correctly selected tests, including the common blood test, the urinalysis, the X-ray and the electrocardiogram (ECG), were highlighted in green. The student missed the spirometry test, which was targeted to determine the patient's respiratory insufficiency, and this missed option was highlighted in yellow. Next, the student can see all results in a form of an actual data from a laboratory: a blood test, a computer tomography (CT) scan of lungs, an ECG (Figure 4). 


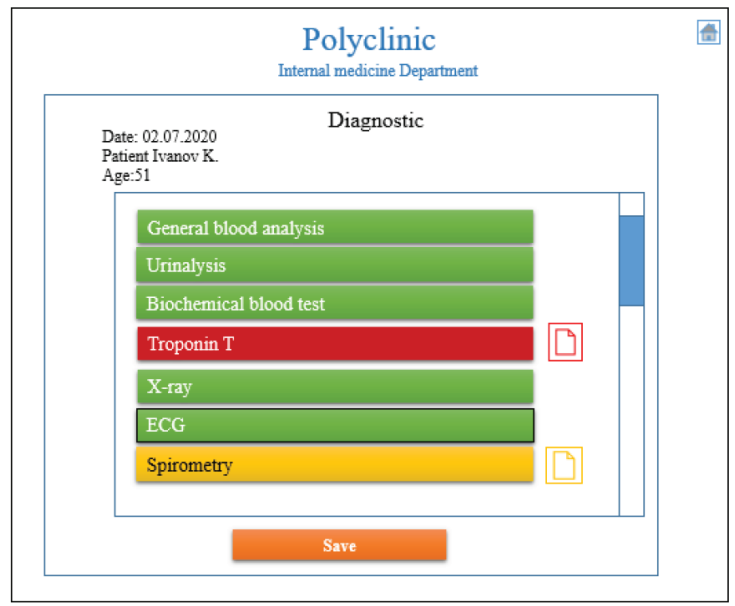

Figure 3 Automatically assessment of the students' choose.

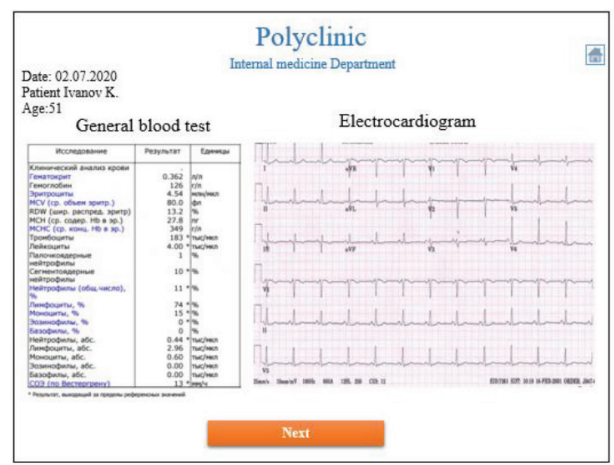

Figure 4 Laboratory and instrumental findings for the patient.

The student should identify existing deviations from normal ranges and mark them. The next step is to consult the patient with a narrow profile specialist(s), which could be selected from a predefined list. The stage ends with creating a clinical diagnosis and recording it into a specially designated field (Figure 5).

Figure 5 demonstrates that the patient's examination revealed an emphysema of lungs on a background of a chronic bronchitis and the type 2 diabetes mellitus. Taking into account the obtained data, the patient needs a complex treatment.

\subsection{Creating a Treatment Program}

On the next stage the student determines the patient's treatment plan. This is a creative process associated with a specialist qualification. An interface of this page has special fields where the student can enter all details of the treatment. There is a field for prescribing medications, a recommended regimen, a diet, and other types of treatment (Figure 6). Therefore, given the long course of the disease, the patient needs an extension of a bed rest and an outpatient supervision. The student prescribes antibiotics, mucolytics, hypoglycemic therapy and, if necessary, hypothermic drugs.

At this stage, the student has an opportunity to refresh the patient information that was collected earlier and store it in the database. The student's job is to select the most appropriate drugs with a selection of an individual dose, method and frequency of using.

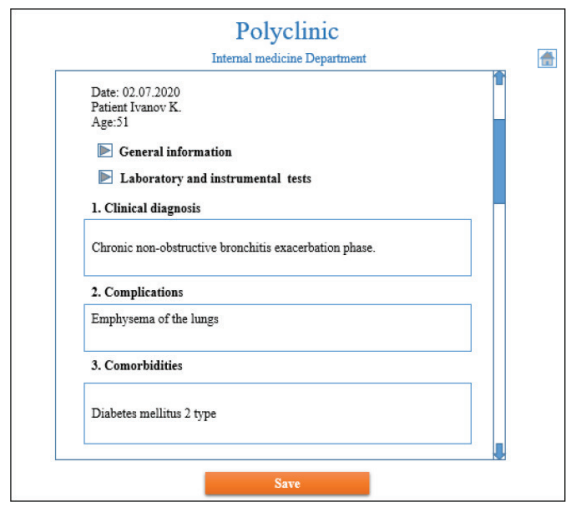

Figure 5 Creating patient's detailed clinical diagnosis.

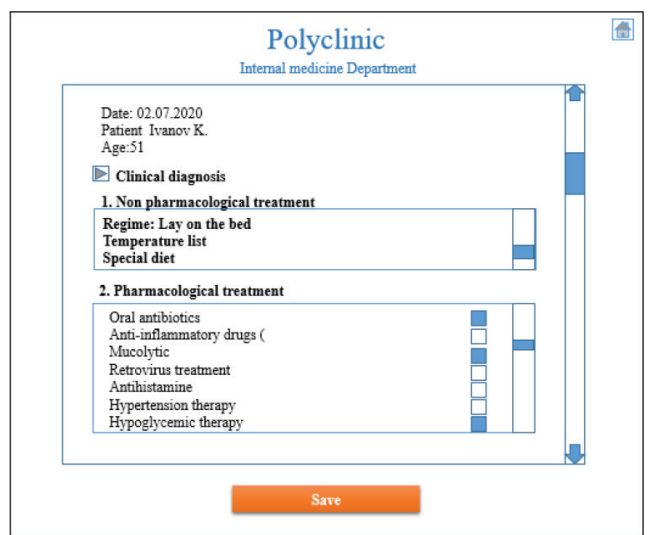

Figure 6 Treatment strategy.

After a treatment is prescribed, a full-fledged EMR is formed for further monitoring of the patient. According to the terms of the task, the student must continue to monitor the patient during the entire period of treatment and conduct daily monitoring of the patient's condition. The program provides the student with questions that he/she could ask the patient. They contain a request about a state of the patient's body temperature, functioning of the main systems and organs etc. After selecting questions, the student receives "patient's" answers, where AI-based program plays a role of the patient.

\subsection{Intellectual System as a Real Patient}

At this stage of solving the case, the AI-based program acts as a patient. During a treatment process, the patient has a number of complications of his condition. For example, an allergic reaction, a delayed stool and gas, an increased blood pressure, etc. The student receives daily information in the form of answers to questions, photos, and audio messages. The program simulates a communication of the student with the patient through typical communication channels, e.g., Telegram, WhatsApp, or Viber. According to our proposed scenario, patient Ivanov K. complained on a headache and a dizziness in the morning on the next day after the first examination. The patient used a home tonometer to measure blood pressure, which showed $160 / 80 \mathrm{~mm} \mathrm{Hg}$. After that, he sent a photo with blood pressure indicators (Figure 7). 


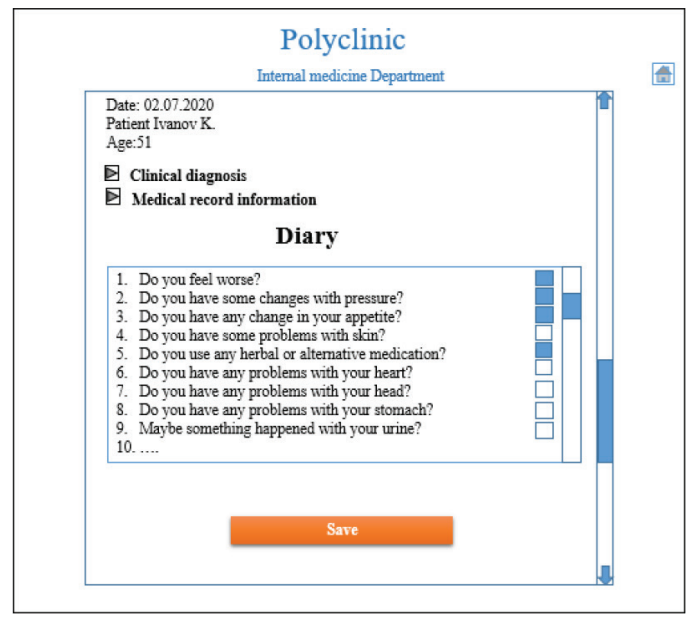

Figure 7 The list of questions for daily observation.

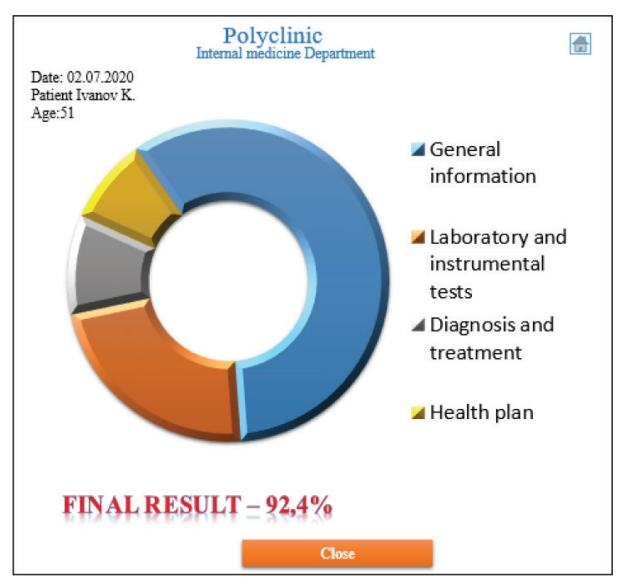

Figure 8 The final result of student's task.

This way the curriculum directs a student in solving daily problems of a patient and there might appear a need to adjust a treatment plan. The script of the case assumes appearance of several pathological symptoms in the patient, which allows a trainee to demonstrate professional skills in solving these problems.

\subsection{Assessment}

Student's actions could be assessed by two methods: automatic (conducted by the program) and expert (conducted manually by a teacher). Based on the prescribed algorithm, the program evaluates a completeness of the patient's examination. If the patient information contains empty spaces, the program will require to complete this work.

The program uses color indicators as follows. Red color indicates an error, yellow indicates deviations from accepted standards, and green indicates the correct actions. The program allows students to improve knowledge using the interactive buttons that appear near the yellow and red fields. After pressing the button, a student can visit a library in the database and read regulatory documents and standards. A final assessment of the work is carried out by a computer taking into account results of the student at all stages of solving the case (Figure 8).

\section{CONCLUSION}

The paper presented a preliminary architecture and an interface for the MIS training program for students of higher medical schools. The program simulates main stages of a polyclinic doctor work in monitoring and treating patients at a pre-hospital stage, which opens up opportunities for independent and distance education. It allows creating EMRs and using a database with regulatory documents for outpatients' treatment. The proposed interface is similar to commercial software products currently used in polyclinics of Kazan city, Russia. The program develops practical skills of medical students in modern approach of EMR processing using artificial intelligence-based support for evaluation of outpatient's conditions, diagnosing a disease and selecting its treatment.

\section{CONFLICTS OF INTEREST}

The authors declare they have no conflicts of interest.

\section{ACKNOWLEDGMENT}

This work was supported by Tatarstan Academy of Sciences, grant ID 20-57-eG.

\section{REFERENCES}

[1] S.L. Schneider, M.L. Council, Distance learning in the era of COVID-19, Arch. Dermatol. Res. 313 (2021), 389-390.

[2] S. Shah, S. Diwan, L. Kohan, D. Rosenblum, C. Gharibo, A. Soin, et al., The technological impact of COVID-19 on the future of education and health care delivery, Pain Physician 23 (2020), S367-S380.

[3] A. Sagitov, H. Li, N. Schiefermeier-Mach, E. Magid, Extending Gazebo simulator for surgical robotics: tissue and suture modeling, in: International Conference on Artificial Life and Robotics, B-Con Plaza, Beppu, Oita, Japan, 2019, pp. 124-127.

[4] H. Lal, D.K. Sharma, M.K. Patralekh, V.K. Jain, L. Maini, Out patient department practices in orthopaedics amidst COVID-19: the evolving model, J. Clin. Orthop. Trauma 11 (2020), 700-712.

[5] E. Magid, A. Zakiev, T. Tsoy, R. Lavrenov, A. Rizvanov. Automating pandemic mitigation, Adv. Robot. 35 (2021), 572-589.

[6] C. Paxton, A. Niculescu-Mizil, S. Saria, Developing predictive models using electronic medical records: challenges and pitfalls, AMIA Annu. Symp. Proc. 2013 (2013), 1109-1115.

[7] M. Samadbeik, F. Fatehi, M. Braunstein, B. Barry, M. Saremian, F. Kalhor, et al., Education and Training on Electronic Medical Records (EMRs) for health care professionals and students: a scoping review, Int. J. Med. Inform. 142 (2020), 104238.

[8] S.I. Karas, I.O. Korneva, M.B. Arzhanik, O.L. Semenova, E.V. Chernikova, O.V. Urneva, et al., The role and prospects of information and communication technologies in developing of physician competencies, Doctor Inform. Technol. 4 (2018), 46-58.

[9] E. Rouf, H.S. Chumley, A.E. Dobbie, Electronic health records in outpatient clinics: perspectives of third year medical students, BMC Med Educ 8 (2008), 13. 
[10] N.M. Pageler, C.P. Friedman, C.A. Longhurst, Refocusing medical education in the EMR era, JAMA 310 (2013), 2249-2250.

[11] D.R. Cheng, T. Scodellaro, W. Uahwatanasakul, M. South, An electronic medical record in pediatric medical education: survey of medical students' expectations and experiences, Appl. Clin. Inform. 9 (2018), 809-816.

[12] A. Rajaram, Z. Hickey, N. Patel, J. Newbigging, B. Wolfrom, Training medical students and residents in the use of electronic health records: a systematic review of the literature, J Am Med Inform Assoc 27 (2020), 175-180.

[13] C. Mountain, R. Redd, C. O'Leary-Kelly, K. Giles, Electronic medical record in the simulation hospital: does it improve accuracy in

\section{AUTHORS INTRODUCTION}

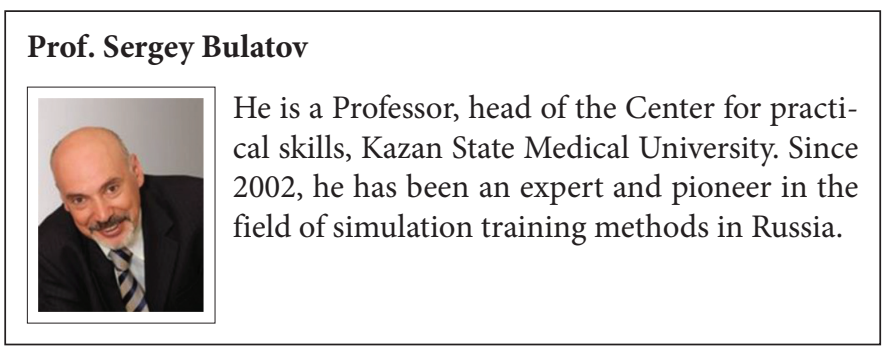

\section{Dr. Enzhe Kharisova}

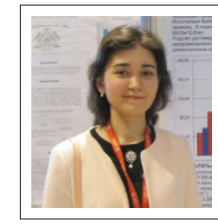

She took her PhD degree in cardiology field. Currently she is a research associate of the Center for practical skills in Kazan State Medical University and also works as a practicing cardiologist.

\section{Dr. Roman Lavrenov}

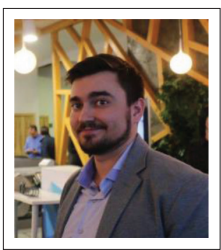

$\mathrm{He}$ is a senior lecturer in Kazan Federal University. He obtained his $\mathrm{PhD}$ at Kazan Federal University in 2020. His main interests are path planning, robot operating system, and autonomous mobile robots. charting vital signs, intake, and output? Comput Inform Nurs 33 (2015), 166-171.

[14] C.M. Yuan, D.J. Little, E.S. Marks, M.A. Watson, R. Raghavan, R. Nee, The electronic medical record and nephrology fellowship education in the united states: an opinion survey, Clin. J. Am. Soc. Nephrol. 15 (2020), 949-956.

[15] S. Bulatov, E. Magid, E. Kharisova, R. Lavrenov, V. Dudin, A. Khazetdinov, Architecture of a student training computer program for preparing professional outpatient consulting skills within an electronic medical records system during COVID-19 alertness situation, in: International Conference on Artificial Life and Robotics, Online, 2021, pp. 36-39.

\section{Prof. Evgeni Magid}

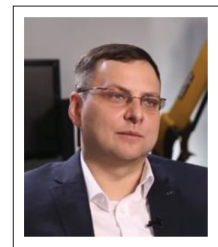

$\mathrm{He}$ is a head of the Intelligent Robotics Department of Kazan Federal University, Russia. He obtained his $\mathrm{PhD}$ degree at the University of Tsukuba, Japan in 2011. His main interests are urban search and rescue, swarm and mobile robotics, and robotics education. 